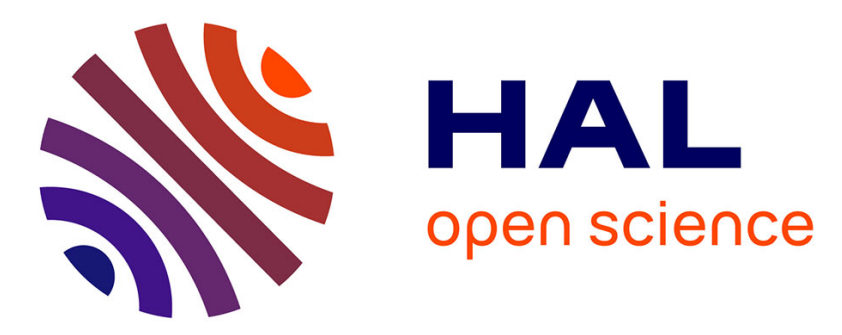

\title{
Espaces de coworking et fablabs des villes petites et moyennes : un rôle de middleground?
}

\author{
Anne-Laure Le Nadant, Clément Marinos
}

\section{To cite this version:}

Anne-Laure Le Nadant, Clément Marinos. Espaces de coworking et fablabs des villes petites et moyennes: un rôle de middleground?. Innovations - Revue d'économie et de management de l'innovation, 2020, 1 (61), pp.41-66. hal-02361833

\section{HAL Id: hal-02361833 \\ https://hal.science/hal-02361833}

Submitted on 19 Nov 2019

HAL is a multi-disciplinary open access archive for the deposit and dissemination of scientific research documents, whether they are published or not. The documents may come from teaching and research institutions in France or abroad, or from public or private research centers.
L'archive ouverte pluridisciplinaire HAL, est destinée au dépôt et à la diffusion de documents scientifiques de niveau recherche, publiés ou non, émanant des établissements d'enseignement et de recherche français ou étrangers, des laboratoires publics ou privés. 


\title{
ESPACES DE COWORKING ET FABLABS DES VILLES PETITES ET MOYENNES : UN RÔLE DE MIDDLEGROUND?
}

\author{
Anne-Laure Le Nadant et Clément Marinos
}

De Boeck Supérieur | «Innovations »

2019/0 Prépublication | pages I74 à XXVI

ISSN 1267-4982

Article disponible en ligne à l'adresse :

https://www.cairn.info/revue-innovations-2019-0-page-I74.htm

Distribution électronique Cairn.info pour De Boeck Supérieur.

(C) De Boeck Supérieur. Tous droits réservés pour tous pays.

La reproduction ou représentation de cet article, notamment par photocopie, n'est autorisée que dans les limites des conditions générales d'utilisation du site ou, le cas échéant, des conditions générales de la licence souscrite par votre établissement. Toute autre reproduction ou représentation, en tout ou partie, sous quelque forme et de quelque manière que ce soit, est interdite sauf accord préalable et écrit de l'éditeur, en dehors des cas prévus par la législation en vigueur en France. Il est précisé que son stockage dans une base de données est également interdit. 


\title{
Espaces de coworking et fablabs des villes petites et moyennes : un rôle de middleground ?'
}

\author{
Anne-Laure LE NADANT \\ Université Rennes 2 \\ Laboratoire LiRIS (EA7481) \\ anne-laure.lenadant@univ-rennes2.fr \\ Clément MARINOS \\ Université Bretagne Sud \\ Laboratoire LEGO (EA2652) \\ clement.marinos@univ-ubs.fr
}

\section{RÉSUMÉ}

L'article interroge la notion de middleground, comme intermédiaire entre l'underground et l'upperground (Cohendet et al., 2010), appliquée au contexte spécifique des villes petites et moyennes. Nous cherchons à savoir dans quelle mesure le modèle des grounds est pertinent pour ces territoires et s'il permet de révéler le fonctionnement des espaces de travail collaboratifs. Les résultats reposent sur une analyse de cas multiple réalisée à partir d'une enquête par entretiens semi-directifs. Ils montrent certaines particularités qui n'ont pas été mises en évidence par la littérature comme un faible cloisonnement entre acteurs (multi-appartenance communautaire, mobilité interstrate) et un fonctionnement ouvert et transversal des espaces étudiés. En conclusion, les différentes strates dans les villes petites et moyennes semblent plus difficiles à distinguer que dans les métropoles.

MOTS-CLÉS : Villes créatives, Villes petites et moyennes, Espaces de coworking, Travail collaboratif

CODES JEL : O18, P25, R58

1. Cet article est issu du projet de recherche INTIMIDE s'intéressant aux tiers-lieux et a bénéficié d'un cofinancement du GIS M@rsouin et de la Maison des Sciences de l'Homme en Bretagne (MSHB). 


\section{ABSTRACT \\ Coworking Spaces and Fablabs in Small and Medium-Sized Cities: Playing the Part of Middleground?}

This contribution questions the notion of middleground as intermediate between the underground and upperground (Cohendet et al., 2010), applied to the specific context of small and medium-sized cities. The aim is to determine to what extent the "ground model" is relevant for such territories and whether it reveals how collaborative spaces work. The results are based on a multiple case analysis based on a semi-directive interview survey. They reveal some particular features that have not been highlighted in the literature, such as a weak fragmentation between actors (multi-community membership, inter-layer mobility) and an open and transversal functioning of the spaces studied. To conclude, the three different layers in small and mediumsized cities seem more intricate than in metropolises areas.

KEYWORDS: Creative Cities, Small and Medium-Sized Cities, Coworking Spaces, Collaborative Work

JEL CODES: O18, P25, R58

Dix ans après la publication de Simon (2009) et des dizaines d'articles sur le sujet (Cohendet et al., 2010a ; Grandadam et al., 2013 ; Cohendet et al., 2014 ; Avdikos, 2015 ; Capdevila, 2015 ; Dechamp, Szostak, 2016 ; Lange, Schüssler, 2018, etc.), il semble opportun de réinterroger la notion de middleground pour comprendre les dynamiques urbaines de créativité. Ces travaux de recherche suggèrent que la capacité créative des villes repose sur un processus institutionnel qui connecte un underground d'individus talentueux et un upperground constitué d'institutions publiques et privées formelles (institutions politiques, grandes entreprises, centres de recherche, établissements d'enseignement supérieur, etc.). Cette connexion entre l'underground et les sphères industrielles et commerciales développe la capacité des villes à générer des innovations mais elle ne s'établit pas spontanément (Caves, 2000 ; Hartley, 2005). Les collectifs informels, les individus et les communautés qui constituent l'underground développent des liens avec les acteurs formels de l'upperground par le biais de plateformes intermédiaires qui constituent le middleground. Le middleground fait ainsi le lien entre les deux autres strates du territoire créatif et assure l'enrichissement de la créativité des métropoles et de leurs quartiers créatifs (Simon, 2009).

Dans cet article, nous nous interrogeons sur la pertinence de la notion de middleground appliquée à d'autres tissus urbains, comme les villes petites et moyennes (VPM) qui restent des territoires peu étudiés dans la littérature (Demazière, 2014). Précisément, les travaux sur le middleground offrent une 
grille pour l'analyse des lieux de création et de transfert de connaissances, comme les espaces de travail collaboratifs. L'objectif de cet article est ainsi, à partir de cette grille, de comprendre le rôle et les fonctions des espaces de coworking (ECW) et des fablabs des VPM et d'analyser les relations entre les trois strates de l'upperground, du middleground et de l'underground.

Ce contexte territorial est particulièrement intéressant à analyser car, depuis plusieurs années, de nombreuses mutations économiques et sociales affectent ces territoires (Carrier, Demazière, 2012). Le périurbain et la périphérie des métropoles changent progressivement de visage. De nouvelles populations, souvent originaires ou ayant une expérience des grands centres urbains, se regroupent et s'agrègent avec les populations existantes dans des lieux de travail dédiés aux rencontres, aux collaborations et à la créativité (Jamal, 2018 ; Aubouin, Capdevila, 2019). On recense aujourd'hui des ECW et fablabs dans de nombreux territoires, y compris ceux qui ne sont pas désignés, de prime abord, comme créatifs. Cette tendance ne concerne pas uniquement les métropoles et les dynamiques de création sont plutôt en faveur des VPM dont la population est inférieure à 200000 habitants (Deskmag.com, 20182). En France, la moitié des ECW dénombrés sont implantés hors métropole (Levy-Waitz et al., 2018). La pratique socio-professionnelle du coworking dépasse largement les frontières des grands centres urbains, notamment grâce au développement des outils et réseaux numériques et à l'arrivée de nouveaux travailleurs dans des territoires jusqu'à présent plutôt voués aux activités plus traditionnelles comme l'industrie ou l'agriculture (Besson, 2017).

Les tiers-lieux, dont font partie les ECW et fablabs, seraient à l'origine un processus urbain, fruit de la ville créative (Moriset, 2014). La plupart des recherches sur ces espaces privilégient les métropoles en négligeant les autres tissus urbains (Azam et al., 2015 ; Capdevila, 2015 ; Liefooghe, 2016 ; Ferchaud, 2018 ; Aubouin, Capdevila, 2019). Pourtant, on assiste, depuis dix ans, à une diffusion des pratiques hors des zones les plus denses et il semble aujourd'hui essentiel de prolonger les analyses sur d'autres strates urbaines.

Dans cet article, nous étudions les différents rôles de trois ECW et d'un fablab situés dans trois VPM du grand ouest français. Les résultats confirment que la notion de middleground conserve son utilité pour comprendre le rôle de ces espaces situés dans ce type de territoire urbain mais la description de son articulation avec l'underground et l'upperground doit être amendée. En effet, dans le contexte territorial spécifique des VPM, nous observons une certaine porosité entre les trois strates, du fait notamment des proximités entre acteurs. Par ailleurs, si les espaces de travail collaboratifs des VPM favorisent

2. Le Global Coworking Survey publié par Deskmag est une étude produite par des acteurs du coworking sur la base de la manifestation spontanée des espaces de coworking eux-mêmes. 
bien la transmission de la créativité de l'underground vers l'upperground, ce rôle semble toutefois secondaire. Ces espaces jouent surtout un rôle social et de mise en réseau, la plupart des acteurs des territoires étudiés n’ayant pas comme objectif principal l'innovation et l'accès au marché.

\section{Revue de la littérature}

\section{Territoires créatifs et villes petites et moyennes}

Selon Dechamp et Szostak (2016, p. 61), « le territoire créatif est défini comme un espace où sont créées diverses connaissances (scientifiques, industrielles mais aussi symboliques), où des idées émergent par et pour les acteurs concernés, c'est-à-dire les organisations, les communautés et les individus, ce qui profite au développement des activités territoriales ». Cordobes et Ducret (2010) ajoutent que le territoire créatif se caractérise par sa population qui, quelle que soit la fonction, la place, l'origine des individus qui la composent, partage une propension à être créatif. La créativité d'un territoire dépendrait ainsi d'une attitude globale de la population à acquérir, développer des comportements marqués par l'expérimentation, l'entreprise, l'innovation, l'échange.

Cette définition semble intrinsèquement liée à celle de la ville créative comme mode de développement urbain visant à faire entrer la ville dans l'économie de la connaissance et de l'innovation (Liefooghe, 2010). Même si leur définition reste ambiguë (Santamaria, 2000), les VPM pourraient, comme les métropoles, constituer des supports de création de connaissances et d'émergence d'idées nouvelles et ainsi être étudiées comme des territoires créatifs. En effet, la seule présence d'acteurs créatifs sur le territoire n'influence pas nécessairement les organisations, communautés et individus. Il est important de créer des espaces d'échanges et des liens favorables à la réunion entre ces acteurs (Dechamp, Szostak, 2016). En particulier, des espaces dédiés aux échanges d'idées et propices à l'émergence d'idées nouvelles sont indispensables pour développer la créativité d'un territoire (Suire, 2013, 2016). Les espaces de travail collaboratifs, qui se sont aussi développés dans les VPM peuvent remplir cette fonction d'intermédiarité (Le Nadant et al., 2018).

D'une manière plus générale, selon des travaux en management, les territoires créatifs sont constitués de trois strates en interaction, composées d'individus et/ou d'organisations : l'upperground, l'underground et le middleground (Cohendet, Zapata, 2009 ; Simon, 2009 ; Cohendet et al., 2010a, 2011). Une ville créative repose ainsi sur un système vertueux composé de ces trois strates. 


\section{Trois strates des territoires créatifs et espaces de travail collaboratifs}

Les trois strates des territoires créatifs ont principalement été étudiées dans le contexte des villes créatives, qui renvoient elles-mêmes au paradigme de la métropolisation. Cela interroge, en creux, l'attribut de ville créative aux seules métropoles. En effet, l'étude des collectifs créatifs et de la classe créative, composés des professions intellectuelles, des artistes et des travailleurs du savoir en général, s'avère relativement peu présente dans la littérature sur les VPM et il en va de même pour les intermédiaires d'innovation (Jamal, 2018).

Selon Simon (2009), « les collectifs créatifs du middleground assument une fonction d'intégration des connaissances et de transfert entre l'underground et l'upperground ». Ils constituent en ce sens une des strates de l'innovation créative (Cohendet et al., 2010a). L'underground est défini par Simon (2009) comme "un ensemble d'activités créatives, artistiques et culturelles, qui se déploient hors des réseaux formellement organisés d'institutions de production, d'exploitation et de diffusion ». Le middleground recouvre donc des plateformes collectives intermédiaires entre l'underground, la strate des processus créatifs non structurés, et l'upperground c'est-à-dire la strate des firmes et des institutions structurées permettant une mise sur le marché des idées créatives.

Le middleground permet la structuration d'activités sociales, économiques et la construction d'espaces cognitifs qui contribuent à la dynamique des activités créatives, participent à la circulation permanente des connaissances entre l'underground et l'upperground, favorisent l'interaction entre les communautés et le développement de la capacité créative de la ville. Le middleground permet aux idées d'émerger, de circuler, d'être développées, enrichies, affinées et valorisées, et finalement de nourrir la capacité d'innovation des acteurs formels. La figure 1 synthétise l'organisation en strates d'un territoire créatif. 


\section{Figure 1 - Les trois strates de l'innovation créative}

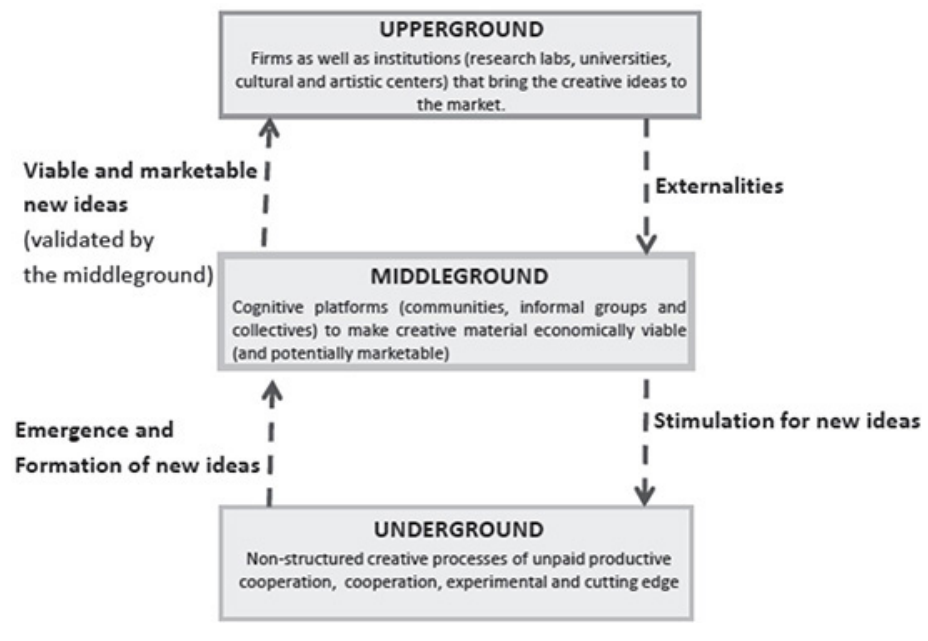

Source : Suire (2013), d'après Cohendet et al. (2010a)

Sarazin et al. (2017, p. 25) affirment que le middleground est un bien commun, co-créé par les membres de la communauté (l'underground) et les organisations qui l'entourent (l'upperground), et caractérisé par quatre composantes complémentaires : des spaces où se développent des échanges construits d'idées ; des places qui permettent aux individus de se rencontrer et de socialiser les expériences ; des événements qui ouvrent le monde local aux influences extérieures; des projets qui amènent les communautés à interagir et travailler ensemble.

Les spaces sont les espaces cognitifs qui orientent les recherches et questionnements et structurent les débats entre les membres de la communauté. Ces espaces cognitifs permettent aux membres de la communauté d'échanger sur les thèmes choisis avec d'autres parties distantes de la communauté ou encore avec d'autres communautés. Les places sont des lieux où les membres peuvent se rencontrer de manière informelle et à l'improviste (Sarazin et al., 2017, p. 25). Il peut s'agir de lieux physiques comme des tiers-lieux, des fablabs, des ECW ou éventuellement virtuels (forums, wikis, etc.). Les membres d'une communauté se retrouvent lors d'événements organisés régulièrement où les membres partagent des moments forts, s'ouvrent à l'extérieur et rencontrent de nouveaux membres. Enfin, les membres les plus impliqués commencent des projets dans lesquels ils s'engagent à concrétiser ensemble les idées émises par la communauté. Ainsi, ces projets valident les contributions et la valeur des connaissances et des idées ayant émergé des rencontres entre les membres d'une ou plusieurs communautés. 
Dans le middleground, la dynamique de l'innovation privilégie, de plus en plus, la mise en œuvre de pratiques et d'expériences ouvertes et informelles. Des formes diverses d'économie collaborative se développent dans de nombreux secteurs et des espaces nouveaux de création collective émergent dans tous les territoires, que ce soit sous forme d'ECW ou encore de fablabs. La littérature sur les espaces de travail collaboratifs montre qu'il existe une grande diversité dans les caractéristiques des lieux (Capdevila, 2015 ; Fabbri, Charue-Duboc, 2016 ; Gandini, 2015 ; Liefooghe, 2016 ; Michel, 2018a ; Suire, 2016). Cependant, les travaux récents ont permis de préciser la définition et la typologie de ces espaces. Scaillerez et Tremblay (2017) distinguent les types d'espaces selon les acteurs qui les composent : le fablab est un "lieu de mise en place d'expériences et d'échanges de pratiques entre fabricants " tandis que l'ECW est un «lieu d'échanges et de partage, une communauté de réseautage ».

D'après Merkel (2015), les ECW agissent comme des interfaces entre les milieux créatifs de la ville et au-delà. Les ECW et fablabs peuvent être considérés comme faisant partie du middleground, à l'intersection de multiples strates et en médiation entre l'underground des individus créatifs et l'upperground des entreprises et des organisations (Suire, 2013, 2016 ; Capdevila, 2015). La rencontre de communautés différentes nécessite des espaces pour connecter les communautés. En particulier, les ECW joueraient un rôle de support d'autant plus important à l'entrepreneuriat et à l'écosystème d'innovation au sein des villes moyennes par leur soutien aux entreprises émergentes, par les réseaux sociaux qu'ils génèrent et par leur capacité à attirer des nouvelles populations (Jamal, 2018).

\section{Question de recherche}

Nous nous intéressons aux collectifs du middleground situés hors métropoles en étudiant le rôle et les fonctions des espaces de travail collaboratifs des VPM à partir des quatre composantes clé décrites par Cohendet et al. (2010a). Nous émettons l'hypothèse que les espaces de travail collaboratifs sont un middleground et qu'ils assument une fonction d'intégration des connaissances et de transfert entre l'underground et l'upperground.

Selon Simon (2009), « il conviendrait de documenter plus finement les modes d'interaction et d'articulation entre les collectifs créatifs et les firmes, dans une perspective plus économique ». Suivant cette perspective, deux questions complémentaires viennent guider nos réflexions :

- La notion de middleground est-elle pertinente pour les VPM ? Les proximités géographiques et organisationnelles (Torre, 2014) entre acteurs pourraient, en effet, modifier l'organisation des dynamiques urbaines de la 
créativité et l'articulation entre les trois strates de l'underground, l'upperground et du middleground.

- Dans quelle mesure le rôle des acteurs du middleground est-il différent dans les VPM par rapport à celui joué dans les métropoles?

Il s'agit donc de confronter la notion de middleground aux résultats d'une enquête menée auprès de quatre espaces collaboratifs situés dans trois VPM, considérés a priori comme des intermédiaires entre l'underground et l'upperground et de mettre en évidence la particularité de leurs rôles et fonctions.

\section{Méthodologie}

Afin d'analyser le fonctionnement des espaces de travail collaboratifs des VPM à partir de la notion de middleground, nous avons réalisé une étude de cas instrumentale multiple (Stake, 1995) qui consiste à circonscrire un phénomène de portée générale à partir de plusieurs situations de ce phénomène.

Les données primaires (Tableau 1) sont issues d'une enquête qualitative par entretiens semi-directifs approfondis menés en 2016 et 2017 auprès de quinze parties prenantes (fondateurs-animateurs et membres) de quatre espaces de travail collaboratifs implantés dans deux villes moyennes et une petite ville (définitions INSEE) : Lorient et Caen (200 000 habitants) et Auray (10 000 habitants). Aucun des espaces étudiés ne pratique la sélection à l'entrée, contrairement à de nombreux espaces implantés dans les grands centres urbains, ce qui permet un certain degré de diversité parmi les membres. Les membres interviewés se sont portés volontaires pour participer à l'enquête, suite à une proposition des animateurs et gestionnaires.

Ce matériau a été complété par plusieurs visites des lieux, des participations aux événements organisés et par les informations disponibles sur les sites internet des espaces. Précisons que les entretiens avec les fondateurs ont été les plus importants pour notre étude afin de comprendre le positionnement de l'espace collaboratif dans son environnement et ses relations avec les acteurs locaux. Nous faisons donc implicitement l'hypothèse que les fondateurs-animateurs bénéficient d'une vision transversale de leur structure. Les entretiens avec les membres ont davantage été utilisés à titre confirmatoire et pour recueillir des témoignages concrets sur les diverses situations de collaboration. Leur faible nombre peut toutefois être considéré comme une limite méthodologique de notre recherche. 
Tableau 1 - Synthèse des données primaires

\begin{tabular}{|c|c|c|c|}
\hline Acteurs & $\begin{array}{l}4 \text { fondateurs-animateurs } \\
\text { ( } 3 \text { femmes et } 1 \text { homme) }\end{array}$ & $\begin{array}{c}11 \text { membres (2 femmes et } 9 \\
\text { hommes) }\end{array}$ & $\begin{array}{c}\text { Total } \\
15\end{array}$ \\
\hline $\begin{array}{l}\text { Professions } \\
\text { (tranche d'âge) }\end{array}$ & $\begin{array}{l}\text { Ancien ingénieur chef de } \\
\text { projet ( } 35-45) \text {, ancienne } \\
\text { responsable d'un centre } \\
\text { nautique (25-35), ancienne } \\
\text { chargée de communication } \\
\text { (25-35), ancienne chargée } \\
\text { de mission (25-35). }\end{array}$ & $\begin{array}{l}\text { Artiste culinaire (25- } \\
\text { 35), professeur (35-45), } \\
\text { architecte (35-45), infor- } \\
\text { maticien ( } 35-45) \text {, chef } \\
\text { d'entreprise (35-45), } \\
\text { juriste (25-35), techni- } \\
\text { cien nautique (35-45), } \\
\text { chefs de projet (25-35), } \\
\text { startupper ( } 25-35), \\
\text { consultante (35-45). }\end{array}$ & - \\
\hline $\begin{array}{l}\text { Durée moyenne } \\
\text { des entretiens }\end{array}$ & $1 \mathrm{~h} 30$ & $1 \mathrm{~h}$ & $17 \mathrm{~h}$ \\
\hline $\begin{array}{l}\text { Thèmes abor- } \\
\text { dés }\end{array}$ & $\begin{array}{l}\text { - Profil, parcours, motiva- } \\
\text { tions } \\
\text { - Nature des relations } \\
\text { entretenues avec les acteurs } \\
\text { locaux extérieurs à l'espace, } \\
\text { notamment les partenaires } \\
\text { ayant soutenu le projet } \\
\text { et ceux avec lesquels les } \\
\text { collaborations sont les plus } \\
\text { abouties. }\end{array}$ & $\begin{array}{l}\text { - Profil, parcours } \\
\text { - Réseaux de contacts } \\
\text { mobilisés dans le cadre } \\
\text { de leur activité (internes } \\
\text { ou externes à l'espace et } \\
\text { antérieurs ou postérieurs } \\
\text { à leur installation). }\end{array}$ & - \\
\hline
\end{tabular}

Le choix des espaces étudiés s'est opéré principalement à partir de leur lieu d'implantation hors métropole. Notre intuition de départ vient du fait que les proximités géographiques et organisationnelles entre acteurs (Torre, 2014) dans ces territoires de taille modeste pourraient jouer un rôle dans les interactions entre les strates de l'upperground, de l'underground et du middleground. Cet élément a nettement encouragé notre décision d'y mener des investigations. Le critère d'auto-désignation, autrement dit les espaces se revendiquant comme collaboratifs, a également guidé nos choix. Enfin, il faut préciser que ce choix relève aussi d'un opportunisme méthodique (Girin, 1989) consistant à ajuster le dispositif de recherche aux contraintes et opportunités offertes au chercheur, notamment dans leur dimension spatiale.

Nous avons choisi d'étudier trois territoires : 1) La Colloc à Lorient, 2) la Cité de chantier à Colombelles près de Caen, 3) La Fabrique du Loch et l'Argonaute à Auray. Après avoir présenté les territoires étudiés et les acteurs des différentes strates, nous proposons une analyse des cas réalisée à partir d'une grille caractérisant les quatre composantes clé du middleground décrites par Cohendet et al. (2010a) : spaces, places, événements et projets. 


\section{Description des trois territoires et de leurs strates}

\section{Le cas de Lorient : la Colloc}

Avec moins de 60000 habitants, Lorient est une ville moyenne. Cette souspréfecture du Morbihan constitue le centre d'une agglomération de 210000 personnes. Historiquement, l'activité économique était fortement structurée par l'économie maritime et militaire et ses grands donneurs d'ordre publics. À la suite des restructurations économiques liées aux fermetures d'usines et des bases militaires dans les années 1990, le tissu économique a évolué vers davantage de petites entreprises, ce qui s'est traduit par un maillage important de réseaux et clubs économiques (Marinos, 2015).

Emblématique des transformations urbaines et économiques en cours, le quartier lorientais de la Perrière témoigne du mélange des trois strates au sein d'un périmètre géographique réduit. L'underground lorientais y est historiquement très présent : l'avenue de La Perrière était un haut lieu des retours de pêches des marins jusque dans les années 1980, avec une activité nocturne intense. On y retrouve toujours, quelques décennies plus tard, cette intensité avec quelques-uns des cafés-concerts mythiques, et néanmoins alternatifs, de la ville comme Le Cargo Sentimental et le Galion. Des ateliers et des collectifs d'artistes comme Le Lieu Noir Lieu Jaune, qui réunit un bar concert associatif et une galerie d'art, et les Ateliers du bout du monde, un espace de travail artistique, s'y sont aussi installés. Le quartier est même qualifié par certains de "port underground $»^{3}$ avec le mur d'expression libre lorientais dédié au street art et son festival de contre-cultures Unies sont nos Cultures. À l'opposé, l'upperground est aussi bien implanté dans le quartier. L'Agence de Développement Économique du Pays de Lorient (AUDELOR), organe du développement économique gouverné par les élus du territoire, se situe à quelques dizaines de mètres de ces lieux de culture alternative et plusieurs ateliers publicitaires et agences de communication ont choisi de s'y établir. Par ailleurs, l'École des Beaux-Arts dont les locaux sont mitoyens de ceux de l'AUDELOR est aussi installée dans le quartier de la Perrière.

Au cœur de ce quartier et à proximité immédiate de ces acteurs, on retrouve l'un des plus grands espaces de coworking de la région Bretagne. La Colloc, initiative privée sous statut de SAS, occupe un bâtiment de 1400 mètres carrés et accueille chaque jour une centaine de travailleurs. L'une de ses fondatrices explique l'intérêt d'une telle situation géographique : « on est

3. https://www.jolisvoyages.com/2018/03/01/lorient-un-port-underground/ 
dans une avenue qui fait partie des quartiers historiques de la ville, qui a une âme. On croit vraiment en son développement et on n'avait pas envie de se mettre dans un quartier d'affaires, aseptisé ». La Colloc accueille régulièrement des artistes à l'occasion d'expositions et l'un de ses membres permanents, graphiste et photographe de métier, affiche ses œuvres sur les murs de l'ECW. De nombreux échanges, organisés ou spontanés, formels ou informels, ont par ailleurs lieu au détour des sorties dans les nombreux bars et restaurants de l'avenue : " on a des restaurants et des cafés tout autour, l'environnement est favorable aux rencontres, c'est important pour une structure comme la nôtre » (cofondatrice).

La Colloc entretient des liens avec certaines des institutions de l'upperground du territoire, notamment la CCI et l'AUDELOR par l'intermédiaire de formations et d'événements régulièrement organisés dans ses locaux comme les «Matinales de l'entreprenariat » ou les ateliers «Entreprendre et se développer ", lors desquels interviennent experts, banquiers et avocats de la place. « La CCI 56 trouve que la démarche de la Colloc est très intéressante. Ses dirigeants sont venus nous rendre visite et sont revenus. On mutualise des formations pour les entrepreneurs. On a envie de faire des choses intelligentes ensemble " (cofondatrice). En outre, les réunions des clubs de dirigeants d'entreprises comme le Centre des Jeunes Dirigeants et le réseau Initiative du Pays de Lorient se déroulent régulièrement dans l'ECW. La Colloc intervient aussi lors des événements dédiés aux réseaux de dirigeants comme le Quai des Réseaux. Ses cofondatrices définissent elles-mêmes leur projet tant comme un lieu que comme un réseau professionnel et un écosystème ouvert. Les échanges sont fréquents avec les élus locaux : « on a le soutien moral de l'Agglo, on s'entend très bien avec ses différentes structures et services. On travaille en étroite collaboration, on les tient au courant de ce qu'on fait. Ça leur arrive de venir ici. Ils sont tous venus visiter le lieu ». On peut donc considérer que les animateurs d'ECW endossent un rôle de «network entrepreneurs » (Arvidsson, 2007).

Les membres de la Colloc, entrepreneurs et travailleurs indépendants ${ }^{4}$, témoignent eux aussi de cette connectivité avec les milieux économiques locaux. Les événements organisés par l'ECW leur profitent : «j'attends avril parce qu'il va y avoir une formation à la CCI sur la prospection et technique de vente ", déclare un entrepreneur de la Colloc. Selon la fondatrice, la plupart ont occupé une activité professionnelle antérieurement à leur installation à la Colloc et cela les conduit à mobiliser fréquemment leur réseau de contacts. "J'ai travaillé pour de très grandes sociétés, un an chez Bouygues, un an chez Groupama, trois ans pour la BNP. J'ai un assez bon réseau parisien dans

4. La liste détaillée des membres permanents et non permanents et de leur profession est disponible à l'adresse suivante : https://communaute.la-colloc.co 
le domaine du web, du graphisme, etc. » (consultante, membre de la Colloc). Les individus s'autorisent des passages de l'upperground vers la périphérie du middleground. Il est donc nécessaire de prendre en compte les dynamiques des trajectoires professionnelles. Autrement dit, quand on intègre un collectif du middleground en devenant membre d'un ECW, on ne laisse pas son réseau et son carnet d'adresses à la porte. Les travailleurs indépendants rencontrés continuent d'entretenir des relations professionnelles avec leurs anciens employeurs. Il ne semble pas y avoir de rupture. Par ailleurs, d'après sa fondatrice, l'ECW était constitué, en 2017, de 30 \% de télétravailleurs issus de grands groupes internationaux, c'est-à-dire d'individus oscillant entre middleground et upperground.

La Colloc, par l'intermédiaire de ses fondatrices et ses membres, est aussi très impliquée dans le RN165, collectif numérique de Bretagne Sud qui «a pour objectif de rassembler les acteurs numériques du territoire, d'accompagner la transition numérique et de mettre en lumière les initiatives ». L'ECW héberge régulièrement ses assemblées générales et sa fondatrice est membre du bureau de l'association dont le siège se situe dans le quartier de la Perrière. Cette organisation non lucrative traduit elle-même la proximité entre les trois strates sur le territoire en s'efforçant d'impliquer et de faire dialoguer et collaborer des acteurs issus de différents milieux, dont des associations citoyennes et les entreprises locales. À titre d'illustration, l'idée du spectacle "Les Saltimbanques du Numérique » a émergé par les rencontres du réseau. Il implique un collectif d'artistes (la Compagnie Équilibres), avec le soutien des organisations de l'upperground (société Orange, collectivités territoriales) et la participation des collectifs du middleground comme la Colloc. Il semble donc bien difficile dans ces conditions de distinguer une frontière nette entre middleground et upperground : à l'échelle du quartier, on assiste à un maillage de communautés. Trois éléments permettent d'expliquer cet enchevêtrement des strates du territoire.

Premièrement, le parcours des fondatrices et des membres de la Colloc explique une partie de la perméabilité observée entre middleground et upperground. Les individus sont mobiles entre l'un et l'autre. L'une des fondatrices a exercé des fonctions de chargée de mission à la Technopole de Lorient pendant plusieurs années avant de créer l'ECW. Cette expérience lui a permis de construire un carnet d'adresses composé de chefs d'entreprises locales et d'établir des relations avec les institutions publiques (collectivités et opérateurs) et privés (clubs et réseaux économiques) qu'elle sait mettre au service de son projet. Les parcours et les trajectoires des individus apparaissent déterminants pour expliquer les liens entre les différentes strates. La multi-appartenance à des communautés, tant en ce qui concerne les fondateurs d'ECW 
que leurs membres, semble négligée dans la littérature sur le middleground bien qu'elle soit observée sur le terrain. Cette forme d'ubiquité communautaire montre les limites de l'analyse par strates. Elle renvoie à la pratique des slashers qui consiste à multiplier des activités rémunératrices ou non, dans un cadre non exclusivement professionnel correspondant à autant de temps collectifs (Bohas et al., 2018).

Deuxièmement, la forte proximité géographique entre les collectifs des différentes strates et leur co-localisation à une échelle micro-locale expliquent une partie des échanges. Le nombre de lieux est assez restreint pour se rencontrer fortuitement : l'avenue compte une vingtaine de bars et restaurants pour à peine cinq cents mètres de long. Elle attire aussi des individus extérieurs au quartier, assurant ainsi une forme de diversité dans les rencontres. De surcroît, la taille relativement modeste de la ville centre (60 000 habitants) joue un rôle dans la sérendipité et les interconnexions. "Ici, c'est facile de se retrouver, on fait des rencontres intéressantes sans aller très loin » (entrepreneur, membre de la Colloc).

Troisièmement, il existe une convergence des valeurs, qui s'illustre principalement, dans le cas du quartier de la Perrière, par un attachement commun et élevé à ce lieu chargé d'histoire. On constate une volonté partagée de contribuer à la vie du quartier qui a subi plusieurs crises économiques et qui continue de se chercher un avenir passant, comme certains l'affirment, par un début de gentrification. La Perrière est désigné comme le quartier le "plus sympa de la ville » par le journal local Le Télégramme ${ }^{5}$. "C'est un quartier qui bouge, on s'y sent bien » (chef de projet, membre de la Colloc). Un sentiment d'appartenance doublé d'une forte identité serait aussi susceptible de faciliter les collaborations entre acteurs issus des différentes strates.

\section{Le cas de Caen : La Cité de chantier}

Caen est une ville moyenne de 110000 habitants située dans le Calvados. Elle constitue le centre d'une agglomération de 200000 habitants. L'économie caennaise du XX' siècle est particulièrement marquée par une industrie d'ampleur : la Société Métallurgique de Normandie (SMN) installée sur le plateau de Colombelles, cité ouvrière à la périphérie de Caen. Les hauts-fourneaux de la SMN, inaugurés en 1917, emploient jusqu'à 6500 ouvriers en 1974. Les années quatre-vingt-dix voient les piliers de l'industrie locale péricliter. Nationalisée et passée sous le contrôle d'Usinor-Sacilor, la SMN ferme le 5 novembre 1993.

5. https://www.letelegramme.fr/morbihan/lorient/la-perriere-un-quartier-qui-ne-cesse-de-changer-29-10-2018-12119148.php 
Normandie Aménagement, la Société d'Economie Mixte (SEM) de la Communauté urbaine de Caen La Mer, a été créée à la fermeture de la SMN pour concevoir le réaménagement des deux cents hectares de friches industrielles laissées par la fermeture de l'usine. Elle est propriétaire du bâtiment de la Grande Halle et est à l'origine de la démarche de conception de sa reconversion en tiers-lieu. Parallèlement, aux côtés de la Communauté Urbaine de Caen La Mer, la SEM a souhaité préparer, en amont, la reconversion du site de la Grande Halle. Ainsi, elle a fédéré les parties prenantes autour d'une préfiguration du projet final et de ses destinations. Afin de porter le projet de la Grande Halle, le Wip (Work in progress) s'est structuré en association en mai 2016 avec l'idée d'animer un premier tiers-lieu circulaire et citoyen, installé à côté de l'ancien atelier électrique de la SMN. La Cité de chantier, tiers-lieu éphémère construit à partir de containers avec un maximum de matériaux issus du réemploi, a ouvert en septembre 2016. Il s'agit d'un lieu de travail, de vie et d'expérimentation. Ce tiers-lieu en modèle réduit de $250 \mathrm{~m}^{2}$, géré par le Wip, sert de support aux premières initiatives permettant de faire vivre et découvrir le projet Grande Halle, dans une dynamique de chantier ouvert.

Le Wip a été créé par cinq membres fondateurs, gestionnaires de l'association, qui, malgré leurs parcours différents, partagent la volonté commune de voir le projet Grande Halle aboutir. En 2017, les douze membres résidents exercent leur activité dans des secteurs différents : paysagiste, négociante en vin, artiste culinaire, développeur web, graphiste, graphiste textile, etc. Lappartenance des fondateurs et résidents du Wip à de nombreux réseaux locaux permet un fort encastrement social du lieu, ce qui constitue un élément fondamental pour la réussite du projet Grande Halle en cours.

L'upperground du territoire créatif caennais est constitué, entre autres, des instances politiques locales (Mairie, Conseil départemental du Calvados, Conseil régional de Normandie), des établissements supérieurs d'enseignement et de recherche (Université de Caen, écoles d'ingénieurs, écoles de commerce, École supérieure d'arts et média, école Brassart, etc.), des pôles de compétitivité locaux (Pôle TES, Mov'eo, Hippolia, Nucléopolis, Novalog), et de quelques grandes entreprises (Renault Trucks, Valeo, Agrial, Robert Bosch Electronique, Carrefour, etc.). La Cité de chantier entretient des liens avec certaines institutions de l'upperground de son territoire. L'un des axes de ce projet de tiers-lieu est de travailler avec les entreprises locales pour lutter contre l'isolement : isolement des personnes très éloignées de l'emploi, des travailleurs indépendants, des chefs d'entreprise, etc. Un club d'entreprises a ainsi été créé pour organiser des rencontres mensuelles entre chefs d'entreprise, des événements communs, pour offrir des services aux salariés comme 
par exemple des afterworks (les Wipéros le jeudi soir). De nombreux événements sont organisés tout au long de l'année, non seulement pour les habitants mais aussi pour les personnes travaillant sur le plateau de Colombelles: des ateliers bien-être et sophrologie, des ateliers cuisine, des soirées de dégustation de vins, des soirées boum soup, etc. Par ailleurs, le Wip a noué des partenariats avec des associations et des établissements d'enseignement. Des animations et événements ponctuels sont ainsi fréquemment organisés à la Cité de chantier en relation avec ces acteurs de l'upperground du territoire, comme par exemple une journée de rencontre avec les habitants de Colombelles organisée autour d'ateliers et d'une disco-soupe par des étudiants de Sciences Po ou encore un atelier de danse avec le directeur du Centre Chorégraphique National de Caen en Normandie.

En parallèle, la Cité de chantier entretient des liens forts avec l'underground du territoire caennais, qui regroupe des individus créatifs de tous types : artistes, inventeurs, étudiants en art, etc. Certains officialisent leur statut de créatif à travers des expositions, leur participation à l'activité d'ateliers de création et du fablab de Caen (le Dôme), des formations validées par un diplôme. La dimension culturelle du projet de la Cité de chantier, qui prend différentes formes, est importante. Le « chantier culturel » du lieu repose sur une programmation construite en mode « chantier en cours » en concertation avec des partenaires culturels locaux. Par ailleurs, la coordinatrice de la Cité de chantier est aussi administratrice d'un théâtre école et son compagnon est comédien. Elle est donc très bien insérée dans le milieu du théâtre vivant, ce qui lui permet d'organiser plus facilement des événements en lien avec le théâtre à la Cité de chantier. Le lieu peut aussi accueillir des compagnies de théâtre pour leurs répétitions. Des concerts sont programmés régulièrement grâce à l'appui d'une association caennaise, Bande de sauvages, qui a notamment une activité de diffusion de concerts. Avec le soutien de la Fondation de France, le Wip organise aussi différents types d'ateliers gratuits et ouverts à tous pour permettre à chacun de " faire » et faciliter la rencontre entre des publics diversifiés : des ateliers graffitis avec le collectif Aéro, des ateliers de construction de mobilier en bois de palette avec l'association Art itinérant ou encore des ateliers de sérigraphie avec l'Encrage. Le volet culturel de la Cité de chantier permet donc d'organiser des rencontres. Ainsi, les résidents peuvent régulièrement rencontrer des gens appartenant à leurs communautés ou bien à d'autres communautés.

Dans le middleground du territoire créatif caennais, nous trouvons le Collectif PAN (regroupant principalement des musiciens), l'Encrage (atelier collectif de sérigraphie), Bazarnaom (collectif de professionnels de différents métiers du spectacle) et les ateliers intermédiaires (espace de création et 
d'exploration des champs artistiques contemporains occupé collectivement par une vingtaine de compagnies de théâtre, de danse et d'artistes visuels). Ces communautés se sont constituées pour regrouper les individus créatifs isolés de l'underground qui n'ont pas d'interactions privilégiées avec l'upperground.

\section{Le cas d'Auray : l'Argonaute et la Fabrique du Loch}

Auray, petite ville de 15000 habitants située dans le Morbihan, compte deux espaces de travail collaboratifs, élément qui mérite d'être souligné pour une ville de cette taille. Elle bénéficie d'une croissance démographique significative qui s'appuie notamment sur sa proximité des deux pôles économiques majeurs du département (Lorient à l'ouest et Vannes à l'est). L'économie de l'agglomération est largement portée par l'industrie touristique avec, à quelques kilomètres, des stations balnéaires réputées comme Quiberon, La Trinité-sur-Mer et Carnac et sa population de travailleurs qualifiés du secteur tertiaire supérieur n'est pas particulièrement importante.

Les deux espaces de travail collaboratifs, l'Argonaute et la Fabrique du Loch, ont été créés en 2016 sous l'impulsion d'un même collectif d'habitants de la ville qui s'est formé au hasard des rencontres, bien qu'il s'agisse de deux structures distinctes. Ces deux lieux sont implantés dans le centre de la ville, ce qui répond aux objectifs de leurs fondateurs d'en faire des lieux de rencontres et d'échanges accessibles et ouverts au plus grand nombre.

La Fabrique du Loch est un fablab hébergeant un petit ECW installé dans l'ancien hospice d'une rue commerçante. En 2017, elle rassemble 200 membres ${ }^{6}$ issus de divers milieux sociaux et professionnels. Plusieurs dizaines d'individus y viennent régulièrement pour expérimenter, échanger sur leur projet et partager des moments conviviaux : «tu as tous les métiers, on couvre du jardinier au Bac+10, il y a aussi beaucoup d'indépendants qui sont dans le web, dans le design ", « la particularité est d'être ouvert à tous » (fondateur de la Fabrique du Loch). Le lieu se caractérise par son ouverture aux autres organisations du territoire comme les milieux scolaires et les associations culturelles et sociales comme la Maison d'Animation et des Loisirs de la ville. Cette diversité se manifeste notamment par la fréquentation régulière des jeunes gens qui « viennent réparer leurs skateboards, faire des pièces en impression 3D, des Minecraft, des Lego ». Le créneau du mercredi après-midi leur est spécialement réservé. La proximité avec d'autres structures comme le lycée d'art

6. Chargé de clientèle, écrivain, ethnobotaniste, skipper, luthier, artiste clown, enseignant, consultant digital, ... la liste exhaustive des professions représentées dans le fablab en 2017 est disponible sur demande auprès des auteurs. 
de la ville contribue à cette ouverture : « des apprentis ébénistes viennent faire leur propre guitare électrique après leurs heures de cours » (fondateur). Des événements sont régulièrement organisés dans les locaux, avec la perspective d'ouvrir le lieu à une diversité d'acteurs : "il y a une réelle mixité sociale qui ne se retrouve pas toujours ailleurs, notamment dans les fablabs d'université qui ciblent une population qui leur est bien spécifique. La force de la Fabrique du Loch est de mélanger toutes les populations ». Le projet se distingue des fablabs d'entreprise où " tu ne vas avoir que les boîtes qui vont avoir accès, c'est une erreur » (fondateur).

Les milieux économiques sont également bien représentés dans le fablab, « on doit avoir une quinzaine ou vingtaine d'autoentrepreneurs et deux SARL " (fondateur de la Fabrique du Loch), et le soutien, dès l'ouverture, d'entreprises locales attachées au projet (trois cabinets d'architecte naval réputés). Cette ouverture est aussi géographique avec la participation de membres extérieurs au territoire : «il y a plein de gars qui ont des doctorats. Ils travaillent un peu partout (Paris, etc.), mais sont basés dans le coin. Ils viennent découvrir le lieu d'abord puis certains participent aux projets spécifiques au fablab». Dans la même perspective, "les chefs d'entreprise du territoire sont éclairés et avides de connaissances »(membre architecte). Les institutions de l'upperground, comme la CCI, figurent aussi parmi les partenaires de la Fabrique du Loch : "On les avait invités à l'inauguration. Quand ils sont venus, je leur ai dit que ça faisait six mois qu'on était ouverts et qu'ils ne venaient que maintenant. C'était le nouveau Président de la CCI. Je lui ai demandé si les fablabs ne l'intéressaient pas. Il a répondu qu'il venait de Paris où il en avait monté un et que c'était pour cette raison qu'il était là. Il est donc sensible aux fablabs » (fondateur). La recherche de complémentarité à l'échelle locale constitue l'un des moteurs de la coopération entre le fablab et la chambre consulaire : « on a une start-up qui essaie de sortir d'ici et on l'envoie à la CCI pour tout ce qui est relatif aux statuts et à l'aide à la création. Nous, on va être davantage sur la partie pratique et eux sur la partie liée au suivi des adhérents " (animateur-fondateur). La mixité professionnelle rencontrée dans le fablab est reconnue comme un de ses atouts par ses adhérents : « il y a un garagiste, électricien et câbleur de voitures. Ce n'est pas un type qui a énormément de diplômes. Par contre, c'est quelqu'un d'extrêmement intelligent, ouvert, cultivé et qui invente des choses » (membre designer).

La création du fablab a contribué à impulser d'autres initiatives localement comme le projet d'un café-concert associatif avec un concept de tiers-lieu et l'implantation d'une compagnie de cirque: "d'autres ont vu que c'était possible de faire bouger les choses à Auray » et que leur lieu pouvait servir « de vecteur pour aiguiller des projets sur la ville» (fondateur). 
Le second espace collaboratif d'Auray, l'Argonaute, devait initialement s'installer au-dessus des locaux de la Fabrique du Loch mais le manque d'espace et l'envergure du projet ont contraint les deux fondatrices à rechercher un local plus adapté. Installé dans l'ancienne maison des associations appartenant à la mairie, l'Argonaute accueille une vingtaine d'artisans-créateurs de la région en mettant à leur disposition des ateliers de travail et des espaces communs, auxquels s'ajoutent des coworkers dans le secteur des services. Comme dans le cas du fablab avec lequel les échanges sont fréquents, l'Argonaute rassemble des profils diversifiés issus des métiers d'art (orfèvre, tapissier, céramiste, dessinateur, etc.) mais également des travailleurs indépendants (journalistes, webmaster). Les acteurs de l'upperground sont présents avec la venue régulière d'entrepreneurs et de la responsable du réseau de dirigeantes « Femme de Bretagne ». Les fondatrices ont, par ailleurs, l'ambition de créer leur propre galerie d'art dans les locaux, en lien avec l'Association des Artistes d'Auray. La profession d'artisan d'art implique d'osciller entre le secteur artistique et celui de la production. Ces professionnels trouvent sur place une multitude de ressources utiles à leur projet : consultants web et communication, coach professionnel, photographe, etc. jusqu'au médecin généraliste qui a décidé d'y installer son cabinet. Cette recherche de mixité et de complémentarité se retrouve d'ailleurs dans l'un des slogans de l'Argonaute : «À l'Argonaute, on partage un espace entre indépendants, petites entreprises, associations, artistes et artisans d'art, venez vous mélanger!».

\section{Analyse du rôle de middleground des espaces collaboratifs des VPM}

Nous examinons les différents rôles joués par les espaces situés dans des VPM à partir des quatre composantes clé du middleground décrites par Cohendet et al. (2010a) : spaces, places, events, projects. Cette analyse permettra de vérifier si les rôles des collectifs du middleground identifiés par ces auteurs dans le contexte de l'industrie du jeu vidéo à Montréal sont les mêmes que ceux des espaces situés dans les VPM.

\section{Des « spaces » pour échanger des connaissances}

Les « spaces » sont des espaces cognitifs favorisant les échanges construits d'idées. Selon Ikeda (2004), dans un espace cognitif, les individus partagent de la connaissance et apprennent à se connaître. À l'intérieur de chaque communauté de spécialistes, les membres échangent régulièrement au sujet de leur pratique grâce à un espace cognitif (Cohendet et al., 2010b). Cet espace 
cognitif permet aux spécialistes d'un même domaine de confronter leurs idées, de développer des hypothèses audacieuses et de valider de nouvelles formes de création (Cohendet et al., 2010a). En participant à des projets, les membres du collectif doivent s'attendre à recevoir les questionnements, commentaires et critiques des autres membres et des partenaires extérieurs. Ces retours, qui peuvent être sévères, sont exprimés dans un contexte de bienveillance, par des experts du domaine et deviennent donc une source légitime d'apprentissage et d'amélioration. A la Colloc, de nombreux membres s'installent dans l'espace dans la perspective de pouvoir concrétiser leurs idées et de «tester » leur modèle économique auprès d'un panel d'entrepreneurs plus chevronnés. Des membres travailleurs indépendants expérimentent ensemble la réponse commune à des appels d'offres pour remporter des marchés plus importants et gagner en visibilité.

À la Cité de chantier, une résidente explique qu'elle trouve dans le collectif de la bienveillance et de la complémentarité entre ses propres connaissances et celles des autres membres : "il y a une nana qui a une boîte de vins, donc quand je travaille sur mes dîners dans le noir, création de menus, elle réfléchit avec moi sur l'association mets et vins; il y a un graphiste, c'est lui qui a fait la plaquette, qui a créé mon logo ». La présentation du Wip sur son site web précise que la mission de l'association est de : «créer des environnements où l'on travaille, échange, crée et découvre de la façon la plus libre et ouverte possible, pour révéler et valoriser le potentiel de chacun, tout en faisant participer à la construction de projets communs, fédérateurs et utiles pour le territoire ». Ainsi, dans les différents espaces collaboratifs étudiés, chaque membre donne un peu de son temps au collectif. Le cas du projet de drone de la Fabrique du Loch est particulièrement intéressant pour illustrer l'émergence d'un espace cognitif commun. Un groupe composé d'une dizaine d'individus membres du fablab, et aux compétences complémentaires, s'est formé pour conduire des expérimentations visant le développement d'un projet de drones en réseau afin d'aider les navigateurs professionnels lors de manœuvres délicates. Pour y parvenir, les connaissances de makers du fablab ont été associées à celles d'informaticiens et de spécialistes du modélisme. "On se retrouve à la Fabrique et puis on discute de ça et, au fur et à mesure, on agrège les connaissances " (membre informaticien). L'espace comme support de proximité géographique joue ici un rôle d'activateur de proximité cognitive (Le Nadant et al., 2018).

En définitive, s'engager dans un collectif implique de s'engager à participer à ses activités, à apporter ses idées, son avis, ses connaissances et compétences. Les membres rencontrés ne restent pas passifs. Ils avancent dans leurs projets et actualisent leurs idées pour continuer à contribuer au collectif et pour y légitimer leur place. 


\section{Des « places » pour se rencontrer}

Les «places » sont des espaces physiques, des lieux de rencontre informels permettant de socialiser les expériences. Le cœur de l'activité du collectif est l'exploration de nouvelles façons de faire dans un lieu donné. Dans ce sens, le collectif est un lieu d'expérimentation, un espace protégé au sein duquel chaque membre peut légitimement tenter de nouvelles expériences, avec le soutien de chacun des autres membres. Comme indiqué sur le site web du Wip, la Cité de chantier est « un vrai labo pour expérimenter et développer son projet $»$. Une résidente interviewée, artiste culinaire, explique que le lieu lui offre cette capacité d'expérimentation : "si je veux faire des expériences culinaires, je peux demander et ce sera sûrement accordé si j'anticipe assez ".

L'aménagement du lieu, avec les espaces communs dédiés aux interactions informelles et non planifiées, contribue également à la circulation de connaissances tacites entre membres. Les "places » ont, en outre, un rôle social pour les individus qui n’apprécient pas de travailler de manière isolée : « ça apporte un équilibre personnel », "c'est un lieu collectif, donc on dit bonjour, on se raconte nos histoires, s'il y a des soucis avec les enfants, [...] des fois, ça tempère aussi, on a des problématiques de travail, on aurait besoin de vider notre sac " (résidente de la Cité de chantier). Les places ne sont toutefois pas seulement des lieux de socialisation pour les membres d'une même communauté. Ils sont aussi des lieux dans lesquels les rencontres imprévues avec des membres d'autres communautés ou des habitants du territoire sont possibles : "on essaie d'être le plus ouvert possible, c'est sans doute la singularité de notre lieu » confirme le fondateur de la Fabrique du Loch. Les quatre espaces étudiés partagent la caractéristique d'accueillir un public peu enclin à se rencontrer par ailleurs, dans d'autres circonstances.

\section{Des « événements », ouverts à l'extérieur}

Chacun des espaces étudiés propose régulièrement des événements, tantôt réservés aux membres, tantôt ouverts au public, avec l'objectif, certes, d'offrir une visibilité au lieu mais surtout de créer des liens sociaux et de l'émulation. Ces événements fournissent un espace temporaire où des professionnels se donnent des opportunités de collaborer et de former des communautés de spécialistes (Grandadam et al., 2013). On peut évoquer l'exemple de la participation de l'Argonaute aux Journées Européennes des Métiers d'Art où les œuvres d'une vingtaine d'artistes, dont la moitié n'est pas résidents, sont présentées au public. Chaque semaine, des moments conviviaux, favorisant les échanges réciproques et le transfert de connaissances tacites, sont aussi organisés : cours de sport, jeux, afterwork, déjeuners et dîners participatifs et 
thématiques. Les événements organisés par les différents espaces constituent une opportunité de consolider des liens forts, ceux qui unissent les membres de la communauté, et de générer des liens faibles, ceux qui les relient avec d'autres communautés (Foster et al., 2003). Ils permettent des rencontres souvent imprévues et génératrices d'idées nouvelles entre des membres de communautés locales qui organisent l'événement et les membres des communautés distantes qui viennent participer : "la Cité de chantier aime aussi les rencontres. C'est un lieu de mixité, de diversité, il y a plein d'artistes qui défilent parce qu'ils ont aussi le volet culturel; du coup, je rencontre aussi plein de gens, des gens que je connais déjà ou des gens, en tant que résidente, je les connaissais pas » (résidente artiste). En outre, grâce au soutien de la Fondation de France, la Cité de chantier organise chaque semaine avec des partenaires associatifs locaux des ateliers gratuits et ouverts à tous (jardinage, réparation de petit électro-ménager et de vélos, sérigraphie, etc). Ces ateliers visent à rompre l'isolement et à rendre possible l'inclusion et la mixité sociale en mettant les participants en situation de « faire » et « fabriquer ».

\section{Des « projets " concrétisant les idées des membres}

Lespace, en assurant la co-localisation temporaire des membres de la communauté, fait émerger des projets. Les collectifs des espaces étudiés ont, chacun à leur manière, l'ambition d'explorer de nouvelles pratiques en proposant des façons de faire différentes et d'assurer une forme de mise en commun non marchande des idées, des connaissances, donnant lieu à des projets impliquant ses membres : "tous les jours on a quelqu'un qui arrive avec un projet différent ", s'enthousiasme l'animateur de la Fabrique du Loch tout en reconnaissant "qu'il y a plein de projets non aboutis dans les placards ». La mise en commun peut favoriser, à terme, la collaboration et l'accès à un réseau plus étendu, et éventuellement la mise en place de projets de grande envergure, porteurs de l'identité du lieu. Ainsi, la Cité de chantier rassemble les forces de ses membres résidents - paysagiste, négociante en vin, artiste culinaire, développeur web, graphiste, graphiste textile - autour des différents projets du Wip (inclusion sociale avec des ateliers gratuits et ouverts à tous, chantier culturel avec une programmation croisant différentes formes culturelles, économie circulaire avec le réemploi de matériaux, etc.). A la Fabrique du Loch, lorsqu'un vétérinaire d'Auray a besoin d'une imprimante 3D pour améliorer sa pratique chirurgicale, les membres de la communauté lui proposent de l'aider à modéliser l'os cassé puis à imprimer la plaque de métal qu'il posera ensuite sur l'animal : "Les gens viennent ici se rencontrer, avoir la banane, avoir des projets incroyables. Rien que ça, ça leur donne une motivation ». Plus 
généralement, les habitants de la ville peuvent intégrer le collectif et s'impliquer librement dans des projets proposés par le fablab.

En somme, si on peut estimer que les quatre composantes du middleground caractérisent bien les espaces étudiés, leur rôle dans les processus d'innovation et de mise sur le marché semble mineur. Ils soutiennent davantage la mise en réseau en endossant un rôle social : « l'intérêt que j'y trouve, c'est plutôt de créer un réseau humain et intégrer une communauté " (chef de projet membre de La Fabrique du Loch). De même, une consultante de la Colloc confirme : «j'ai plutôt noué des relations amicales que professionnelles ici ». Cette ancienne parisienne qui ne connaissait personne en s'installant à Lorient s'est ainsi recréée un réseau d'amis grâce à l'ECW. Un autre membre de La Colloc, entrepreneur, avait, dans un premier temps, choisi de travailler de chez lui, puis de louer un appartement. Après quelques années à travailler seul et une courte période de dépression, il se projette maintenant "sans date de fin » comme résident de La Colloc car cela lui a permis de sortir de l'isolement dont il souffrait. Par ailleurs, les membres des espaces partagent régulièrement leur carnet d'adresses: "à force de se fréquenter, on connaît les gens et on peut leur faire confiance pour des recommandations et pour se transmettre des contacts » (membre architecte de la Fabrique du Loch).

\section{Discussion et conclusion}

Au regard de nos résultats, peut-on finalement convoquer la notion de middleground dans le contexte des VPM ? Les trois strates du territoire créatif traduisent une approche relativement « segmentante » mais cette segmentation est difficile à observer dans les espaces étudiés. Cela peut être expliqué par la forte proximité géographique des acteurs et les parcours professionnels des membres des middlegrounds dans le temps. Ces derniers continuent d'interagir avec leur strate d'origine, importent et entretiennent leur capital social et les ressources liées lorsqu'ils intègrent une communauté du middleground. On constate un faible cloisonnement entre acteurs avec un fonctionnement transversal, à l'échelle de la ville ou du quartier, des organisations du middleground. Il est intéressant de signaler que l'ouverture que nous avons pu observer dans les espaces étudiés se retrouve aussi dans les clubs d'entreprises qui rassemblent une grande diversité d'entrepreneurs des VPM car ils n'ont bien souvent, dans ces territoires de dimension modeste, pas la taille critique pour s'organiser en secteur d'activité à part entière (Marinos, 2015). En outre, aucun des espaces étudiés ne pratique la sélection à l'entrée, contrairement à de nombreux espaces implantés dans les grands centres urbains. Cette ouverture, qui pourrait être spécifique aux VPM, autorise un certain degré 
de diversité parmi les membres. Si elle est ouvertement souhaitée et assumée par les animateurs et fondateurs rencontrés, elle semble aussi indispensable compte tenu de la jeunesse des espaces étudiés et de leur implantation dans des territoires relativement peu denses en population, comparés aux métropoles.

Les VPM et leurs espaces collaboratifs constitueraient, par ailleurs, des échelles de proximité où la porosité des frontières (Figure 2) serait renforcée par l'adhésion à des valeurs territoriales communes et le sentiment partagé de suivre le même destin local, autrement dit par l'appartenance à une communauté de destin liée au territoire, à l'échelle d'un quartier ou du centre-ville? La proximité spatiale des VPM renforcerait, plus qu'ailleurs, les interactions sociales (Lugan, 1997). Ce sentiment d'appartenance commun est susceptible de transcender l'adhésion à d'autres formes de communauté et contribuerait à faciliter les échanges entre les différentes strates du territoire créatif.

\section{Figure 2 - Strates et composantes du middleground selon le type de territoire urbain}

Cas de Montréal (Cohendet et al.)

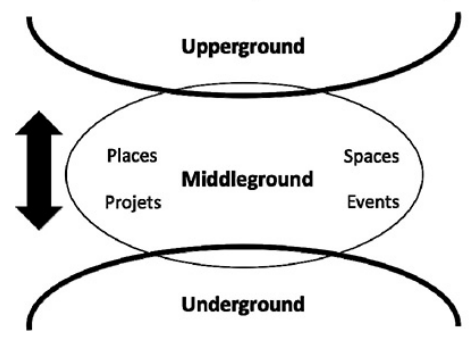

Source: Auteurs
Villes petites et moyennes

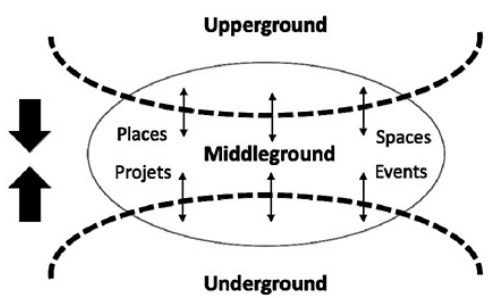

Finalement, malgré certaines limites méthodologiques dues notamment au nombre réduit d'entretiens réalisés, nous avons montré que les quatre composantes clé du middleground se retrouvent bien dans les espaces étudiés. L'activation simultanée des quatre composantes - spaces, places, événements, projets - au sein des ECW et fablabs des VPM assure ainsi des échanges propices à la créativité, au sein des communautés et entre les communautés. En revanche, nos analyses amènent à interroger le fonctionnement des interactions entre les strates appliqué au contexte territorial des VPM. Nos résultats indiquent que la multi-appartenance à des communautés et les nombreux liens qu'elles entretiennent entre elles rendent moins pertinente la représentation de la dynamique de la créativité territoriale en trois strates. Outre

7. Ce phénomène n'est pas spécifique aux VPM. Il a aussi été observé dans les quartiers de grandes villes (voir notamment Michel [2018b]). 
le type de territoire étudié, cette spécificité pourrait provenir de la relative approximation du modèle des strates. En définitive, nos résultats montrent qu'on ne peut pas distinguer aussi clairement les différentes strates dans les VPM étudiées comparées au cas de la métropole montréalaise (Figure 2). Dans les VPM, on constate ainsi une très grande perméabilité entre les trois strates du territoire créatif. Par ailleurs, les bénéfices du middleground des VPM pour l'underground et l'upperground qui l'entourent sont différents de ceux constatés par Cohendet et al. (2010a) dans l'industrie du jeu vidéo à Montréal. Les interfaces entre les trois strates du territoire favorisent bien l'idéation et la circulation des idées mais l'ensemble des acteurs des VPM n'a pas comme objectif premier l'innovation et l'accès au marché. Le rôle de middleground joué par les espaces collaboratifs des VPM est davantage social avec notamment la mise en réseau.

\section{RÉFÉRENCES}

ARVIDSSON, A. (2007), Creative Class or Administrative Class? On Advertising and the 'Underground', Ephemera: Theory $\mathcal{E}$ Politics in Organization, 7(1), 8-23.

AUBOUIN, N., CAPDEVILA, I. (2019), La gestion des communautés de connaissances au sein des espaces de créativité et innovation : une variété de logiques de collaboration, Innovations, 58(1), 105-134.

AVDIKOS, V. (2015), Processes of Creation and Commodification of Local Collective Symbolic Capital: A Tale of Gentrification from Athens, City, Culture and Society, 6(4), 117-123.

AZAM, M., CHAUVAC, N., CLOUTIER, L. (2015), Quand un tiers-lieu devient multiple. Chronique d'une hybridation, Recherches sociologiques et anthropologiques, 46(2), $87-104$.

BESSON, R. (2017), La régénération des territoires ruraux par les Tiers-Lieux. Le cas des Tiers-Lieux Creusois, Urbanews, 18 septembre.

BOHAS, A., FABBRI, J., LANIRAY, P., DE VAUJANY, F. X. (2018), Hybridations salariat-entrepreneuriat et nouvelles pratiques de travail : des slashers à l'entrepreneuriatalterné, Technologie et Innovation, 18(1), 1-19.

CAPDEVILA, I. (2015), Co-working Spaces and the Localised Dynamics of Innovation in Barcelona, International Journal of Innovation Management, 19(3), 1540004.

CARRIER, M., DEMAZIÈRE, C. (2012), Les mutations économiques des villes petites et moyennes, Revue d'Économie Régionale et Urbaine, 2, 135-149.

CAVES, R. E. (2000), Creative Industries: Contracts between Art and Commerce, Cambridge, Mass, Harvard University Press.

COHENDET, P., ZAPATA, S. (2009), Innovation and Creativity: Is there Economic Significance to the Creative City?, Management International, 13, 23-36. 
COHENDET, P., GRANDADAM, D., SIMON, L. (2010a), The Anatomy of the Creative city, Industry and Innovation, 17(1), 91-111.

COHENDET, P., LLERENA, P., SIMON, L. (2010b), The Innovative Firm: Nexus of Communities and Creativity, Revue d'économie industrielle, 129-130, 139-170.

COHENDET, P, GRANDADAM, D., SIMON, L. (2011), Rethinking Urban Creativity: Lessons from Barcelona and Montreal, City, Culture and Society, 2(3), 151-158.

COHENDET, P., GRANDADAM, D., SIMON, L. (2014), Epistemic Communities, Localization and the Dynamics of Knowledge Creation, Journal of Economic Geography, 14(5), 929-954.

CORDOBES, S., DUCRET, R. (2010), Le « territoire créatif » : nouveau modèle ou utopie ?, in Conseil d'analyse économique, Créativité et innovation dans les territoires, Paris, La Documentation française, 327-351.

DECHAMP, G., SZOSTAK, B. (2016), Créativité organisationnelle et territoire créatif : nature de l'influence et enjeux stratégiques pour les organisations, M@n@gement, $19(2), 61-88$.

DEMAZIÈRE, C. (2014), Pourquoi et comment analyser les villes moyennes ? Un potentiel pour la recherche urbaine, Métropolitiques, 29 janvier.

DESKMAG (2018), Global Coworking Survey 2018, http://www.deskmag.com/en/background-of-the-2018-global-coworking-survey-market-research (consulté le 2 avril 2019).

FABBRI, J., CHARUE-DUBOC, F. (2016), Les espaces de coworking : nouveaux intermédiaires de l'innovation ouverte ?, Revue française de gestion, 254, 163-180.

FERCHAUD, F. (2018), Fabriques numériques, action publique et territoire : en quête des living labs, fablabs et hackerspaces (France, Belgique), Thèse de Doctorat, Université Rennes 2.

FOSTER, M., MEINHARD, A., BERGER, I. (2003), The Role of Social Capital: Bridging, Bonding or Both, Working Paper Series, Volume 2003 (1), Toronto, Ryerson University.

GANDINI, A. (2015), The Rise of Coworking Spaces: A Literature Review, Ephemera: Theory $\mathcal{E}$ Politics in Organization, 15(1), 193-20.

GIRIN, J. (1989), Lopportunisme méthodique dans les recherches sur la gestion des organisations, Journée d'étude la recherche action en action et en question, AFCET, collège de systémique, École centrale de Paris, 10 mars.

GRANDADAM, D., COHENDET, P., SIMON, L. (2013), Places, Spaces and the Dynamics of Creativity: The Video Game Industry in Montreal, Regional Studies, 47(10), 17011714.

HARTLEY, J. (2005), Creative Industries, Oxford, Blackwell Publishing.

IKEDA, S. (2004), Urban Interventionism and Local Knowledge, The Review of Austrian Economics, 17(2/3), 247-264.

JAMAL, A. (2018), Coworking Spaces in Mid-Sized Cities: A Partner in Downtown Economic Development, Environment and Planning A: Economy and Space, 50(4), 773788.

LANGE, B., SCHÜßLER, E. (2018), Unpacking the Middleground of Creative Cities: Spatiotemporal Dynamics in the Configuration of the Berlin Design Field, Regional Studies, 52(11), 1-11. 
LE NADANT, A.-L., MARINOS, C., KRAUSS, G. (2018), Les espaces de coworking. Le rôle des proximités dans les dynamiques collaboratives, Revue française de gestion, 44(272), 121-137.

LEVY-WAITZ, P., DUPONT, E., SEILLIER R. (2018), Faire ensemble pour mieux viure ensemble - Mission coworking territoires travail numérique, Rapport du Ministère de la cohésion des territoires.

LIEFOOGHE, C. (2010), La ville créative : utopie urbaine ou modèle économique ?, L'Observatoire, 36(1), 34-37.

LIEFOOGHE, C. (2016), Tiers-lieux, coworking spaces et FabLabs : nouveaux lieux, nouveaux liens et construction de communautés de connaissance créatives, Lille, métropole créative, 183-222.

LUGAN, J.-C. (1997), Sociabilité et intégration dans les petites villes : hypothèses sur une évolution, in Laborie, J.-P, Renard, J. (dir.), Bourgs et petites villes, Toulouse, Presses Universitaires du Mirail, 399-406.

MARINOS, C. (2015), Le développement des entreprises et des territoires en ville moyenne analysé au prisme des réseaux : le cas de la Bretagne Sud, Thèse de Doctorat, Université Rennes 2.

MERKEL, J. (2015), Coworking in the City, Ephemera: Theory $\mathcal{E}$ Politics in Organization, 15(2), 121-139.

MICHEL, B. (2018a), Émergence de dynamiques entrepreneuriales au sein d'espaces de coworking pour entrepreneurs culturels et créatifs, Géographie, économie, société, 20(3), 295-317.

MICHEL, B. (2018b), Mythe et réalités du quartier-village des travailleurs créatifs à Berriat (Grenoble), Territoire en mouvement Revue de géographie et aménagement, 38.

MORISET, B. (2014), Créer les nouveaux lieux de la ville créative. Les espaces de coworking. halshs- 00978718

SANTAMARIA, F. (2000), La notion de "ville moyenne » en France, en Espagne et au Royaume-Uni, Annales de géographie, 109(613), 227-239.

SARAZIN, B., COHENDET, P., SIMON, L. (2017), Les communautés d'innovation : de la liberté créatrice à l'innovation organisée, Paris, Éditions EMS.

SCAILLEREZ, A., TREMBLAY, D.-G. (2017), Coworking, fab labs et living labs, Territoire en mouvement Revue de géographie et aménagement, 34.

SIMON, L. (2009), Underground, upperground et middleground : les collectifs créatifs et la capacité créative de la ville, Management International, 13, 37-51.

STAKE, R. E. (1995), The Art of Case Study Research, Thousand Oaks, Sage.

SUIRE, R. (2013), Innovation, espaces de co-working et tiers-lieux : entre conformisme et créativité, Working Paper, 2013-08, Center for Research in Economics and Management (CREM).

SUIRE, R. (2016), La performance des lieux de cocréation de connaissances : Le cas des FabLabs, Réseaux, 196(2), 81-109.

TORRE, A. (2014), Relations de proximité et comportements d'innovation des entreprises des clusters, Revue française de gestion, 5, 49-80. 